\title{
ADC Child Growth Standards in action
}

ONLINE Stef van Buren and Jacobus $P$ van Wouwe

Arch. Dis. Child. 2008;93;549-551

doi:10.1136/adc.2007.136010

Updated information and services can be found at:

http://adc.bmj.com/cgi/content/full/93/7/549

These include:

References This article cites 12 articles, 2 of which can be accessed free at: http://adc.bmj.com/cgi/content/full/93/7/549\#BIBL

Rapid responses You can respond to this article at: http://adc.bmj.com/cgi/eletter-submit/93/7/549

Email alerting Receive free email alerts when new articles cite this article - sign up in the box at service the top right corner of the article

Notes

To order reprints of this article go to:

http://journals.bmj.com/cgi/reprintform

To subscribe to Archives of Disease in Childhood go to:

http://journals.bmj.com/subscriptions/ 


\section{WHO Child Growth Standards in action}

\section{Stef van Buuren, ${ }^{1,2}$ Jacobus $P$ van Wouwe}

In 1994 the World Health Organization (WHO) began planning for new growth standards. The main motivation came from the observation that clinically significant differences exist between the growth patterns of healthy breastfed infants and the NCHS/WHO international growth references. The WHO recognised that growth references are often used as standards, that is, as tools that enable assessment of how children ought to grow rather than describing how they grew at a particular time and place. A global standard emphasises the notion that all humans are equal. The key assumption underlying the work is that growth is driven by the environment, more particularly, "the biological reality that environmental differences rather than genetic endowments are the principal determinants of disparities in physical growth".

The Multicentre Growth Reference Study (MGRS; 1997-2003) collected data on the growth of 8500 children aged 0 5 years from six sites with various ethnic backgrounds and cultural settings (Brazil, Ghana, India, Norway, Oman and the USA). Only children from privileged, healthy populations were included to reduce the impact of environmental variation. Moreover, their care had to follow recommended practices and behaviours associated with healthy outcomes. The MGRS resulted in the new WHO Child Growth Standards (WHO-CGS), available for height-for-age, weight-for-age, weightfor-height, BMI-for-age, head circumference-for-age, arm circumference-for-age, subscapular skinfold-for-age, triceps skinfold-for-age, and motor development milestones (see http://www.who.int/ childgrowth). Special issues of the Acta Paediatrica, Journal of Nutrition and Food Nutrition Bulletin are dedicated to the WHO-CGS.

\footnotetext{
${ }^{1}$ Netherlands Organization for Applied Scientific Research TNO, Quality of Life, Leiden, The Netherlands; ${ }^{2}$ University of Utrecht, Utrecht, The Netherlands
}

Correspondence to: Professor Stef van Buuren, Netherlands Organization for Applied Scientific Research TNO, Quality of Life, PO Box 2215, 2301 CE Leiden, The Netherlands; stef.vanbuuren@tno.nl
The WHO-CGS are a set of universal standards and as the interpretation of a standard deviation score (SDS) is the same everywhere, will foster crossnational comparison. Moreover, the availability of a global standard facilitates the construction and validation of widely applicable referral and intervention policies that are based upon it. The MGRS study was carefully carried out and extensively documented, and the standards were derived using state-of-the-art statistical approaches. The WHO-CGS are expected to have a major impact on the health and nutrition of children everywhere.

Of course, this does not preclude questions that surround the use of the new standards, and this volume of the Archives of Disease in Childhood contains two articles that raise some of these issues. Hui et $a l^{2}$ write that "these growth standards could be invalid or even misleading for the one fifth of the global population in East Asia" and conclude that "the standards are probably not suitable for Hong Kong Chinese, and by extension for any other infants from East Asia". Wright et $a^{3}$ are more supportive of the new standards but remark that they "may not be simply transferable to the UK".

\section{SITE SELECTION}

Are the WHO-CGS applicable to East Asia? Hui et al present two arguments why this may not be the case. The first relates to site selection, the second to optimal growth.

Hui et al question the usefulness of the WHO-CGS because "the WHO sample did not include infant populations from China". Their concern about site selection is a valid one. Ideally, the sample should be a random selection from the collection of all sites with healthy populations. It is understandable that the WHO decided that each of the six major geographical regions should be represented. ${ }^{4}$ The WHO included just one site from each region, presumably for practical reasons. The criteria for inclusion are documented, and preliminary surveys were held among four candidate sites in Asia. According to the $\mathrm{WHO}$, the final decision to select sites was based on "the results of the surveys, ...the presence of collaborative institutes able to implement the MGRS protocol, and the availability of national and international funds". ${ }^{4}$ The danger of selecting just one site per region is that one could inadvertently exclude a healthy population that grows in a different way from those selected. This danger is real because a previous study carried out by the WHO among breastfed children from seven countries (Australia, Chile, China, Guatemala, India, Nigeria and Sweden) found growth patterns were "strikingly similar" except for India and China. ${ }^{5}$ The authors of that study concluded: "Therefore, children from South and East Asian populations should be represented and their growth rigorously assessed in the process of developing the new international growth reference". Whether this also implies that sites from both India and China must be part of the MGRS is not clear.

\section{Perspective on the paper by Hui et al (see $p$ 561) and Wright et al (see p 566)}

The MGRS combined data from six sites. There is obvious heterogeneity between the sites. The percentage of variance attributable to sites measures the amount of disagreement between the six populations. The WHO estimated this percentage to be $3.4 \%$, and presented this as evidence that the measurements from the six study sites could safely be pooled. ${ }^{6}$ Figure 1 shows that the percentage actually varies by age from $1 \%$ (at 12 months) to $7 \%$ (at 60 months). The

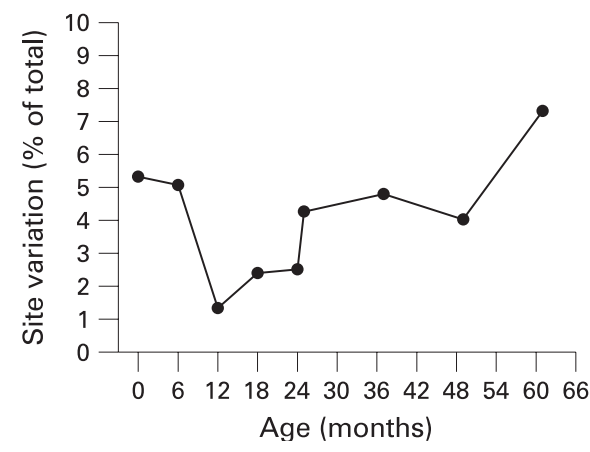

Figure 1 Variation between $\mathrm{WHO}$ sites as a percentage of the total variation for length/ height in the WHO MGRS study at different ages (calculated from table 4 in the article by the WHO Multicentre Growth Reference Study Group ${ }^{6}$ ). 
growth in the various populations is stunningly similar for children aged 12 24 months: children of this age have the same length irrespective of where they live.

The objective of Hui et al is to test the hypothesis that the WHO-CGS "have universal applicability". In order to refute this proposition, differences between local growth patterns and the WHO-CGS need to be substantial. The authors found that Chinese 3-year-old children in an optimal environment are shorter by -0.32 (boys) and -0.36 (girls) SDS than the WHOCGS. This is statistically significantly different from zero, but is it substantial?

Figure 2 plots the average length/height patterns found in the six sites on the $\mathrm{z}$ sites vary approximately between -0.4 and +0.4 SDS. When the data from Hui et al are added to the plot, they fall into the same range. The growth pattern is actually not unlike those from the MGRS sites. The magnitude of the deviations found by Hui et al falls into the range that the WHO considered to be normal site variation. This suggests that including China in the MGRS would have had a relatively small effect on global standards. Some other evidence suggests similar results. ${ }^{7}$ Additionally, the heights of Chinese children have recently reached or even surpassed the WHO-CGS. ${ }^{8}$

\section{OPTIMAL GROWTH}

A second concern of Hui et al is "the WHO assumes and states that under optimal environmental conditions infants and children can achieve their full genetic height potential within one generation, without regard to their parents' size". We believe that Hui et al misquote the WHO score scale. ${ }^{6}$ The average patterns of the

here. In particular, we were unable to find the critical phrase "within one generation" in the cited article, or in any other relevant documents published by the WHO.

The concern of Hui et al calls attention to fact that it is not known whether the statement that physical growth under optimal conditions is independent of time and location is true. The assumption implies that environmental factors are the principal determinants of observed secular and country-to-country variation in growth. An important piece of evidence supporting this theory is that substantial secular increases in stature can occur over just a couple of generations. This does not rule out the possibility that some country-to-country variation is genetic. About $85-90 \%$ of total genetic variability is thought to reside within populations, whereas only $10-15 \%$ can be attributed to differences between populations. $^{9}$

Not all increases in growth are healthy. From a historical perspective it is understandable that nutritional intervention studies have focused on maximal, rather than optimal, growth. However, it is well known that excess weight gain may result in obesity and adverse health outcomes. Also, postnatal height velocity can increase beyond safe levels, high rates being associated in the long term with risks for cardiovascular disease, diabetes and endocrine malignancy. ${ }^{10} 11$ Therefore, safe levels of growth velocity need to be determined. The WHO plans to release such standards.

Many factors that influence growth vary between countries. Examples include gestational age, breast feeding prevalence, nutritional status, physical activity,

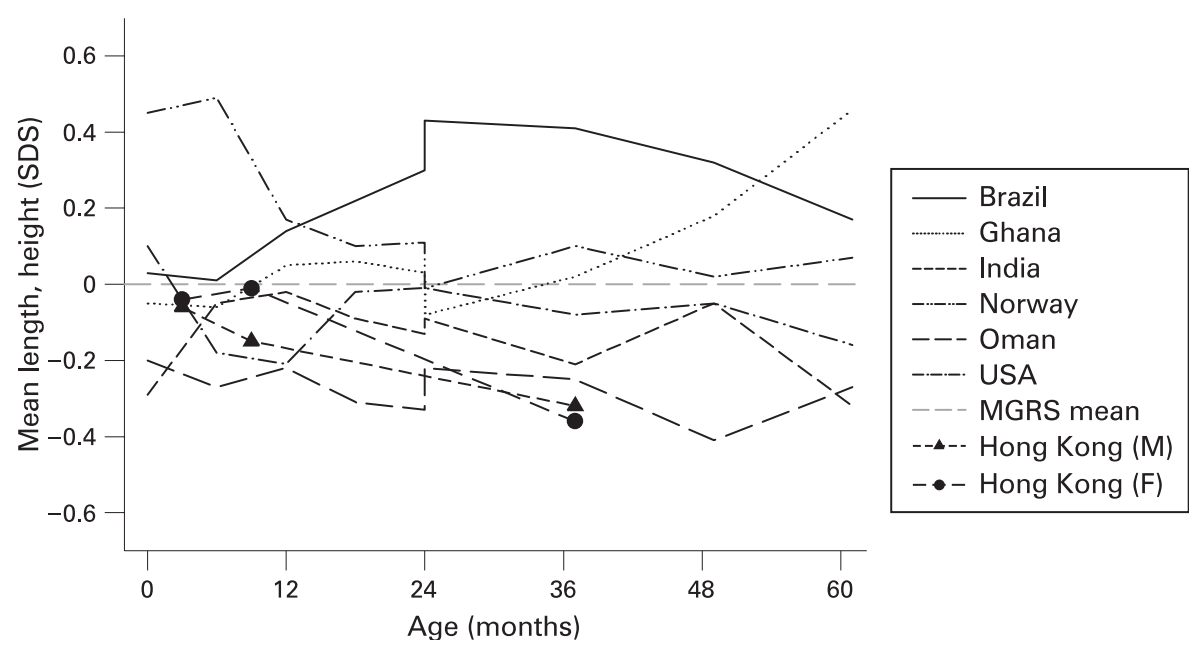

Figure 2 Mean length/height (SDS) for the six study sites from the WHO MGRS study. Data from the Chinese boys and girls from Hui et al are included. ${ }^{2}$

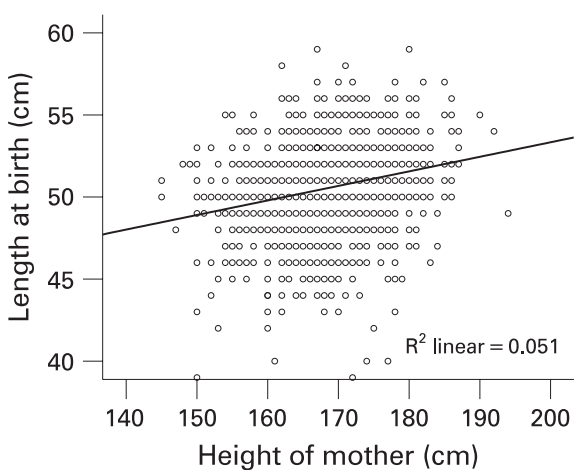

Figure 3 Relationship between infant birth length and height of the mother from the Smock study $(n=2151)$ : birth length $=35.6+0.089$ mother length+error $\left(R^{2}=0.051, p<0.001\right) .^{12}$

wealth and epigenetic interactions between genetic and environmental factors. Many of these factors potentially contribute to inequalities in health and growth, but their relative importance is still unclear. The WHO-CGS provide a natural standard to study this situation.

\section{PREVALENCE AND CUT-OFF VALUES}

Wright et al compare two UK cohorts to the WHO-CGS. The mean length and height of the UK infants were generally close to the WHO-CGS, but UK infants are longer at birth. Wright et al suggest that increased birth length may be due to maternal size. We found in the Smock data $^{12}$ that stature of the mother is indeed related to infant length at birth (fig 3). Thus, the explanation offered by Wright et al seems plausible. It is remarkable that these initial differences at birth diminish until the age of 12 months.

Wright et al show that the WHO-CGS for weight are lower than the UK reference. Unless the WHO publishes the intersite means for weight, it is impossible to assess how the difference relates to normal inter-country variation. Adoption of the standards would substantially alter the UK prevalence of underweight, overweight and weight faltering. This presents problems if continuity of the prevalence series is important. A change in standards can alter the diagnostic characteristics (sensitivity, specificity) of any rules applied to individual growth curves. We think that it is usually possible to avoid this by adapting the cut-off value.

Both Wright et al and Hui et al report standard deviations of the SDS in their populations. Note that these are usually $<1$. This has a statistical explanation. The WHO-CGS combine variation between individuals within sites with heterogeneity between sites. Although the heterogeneity 


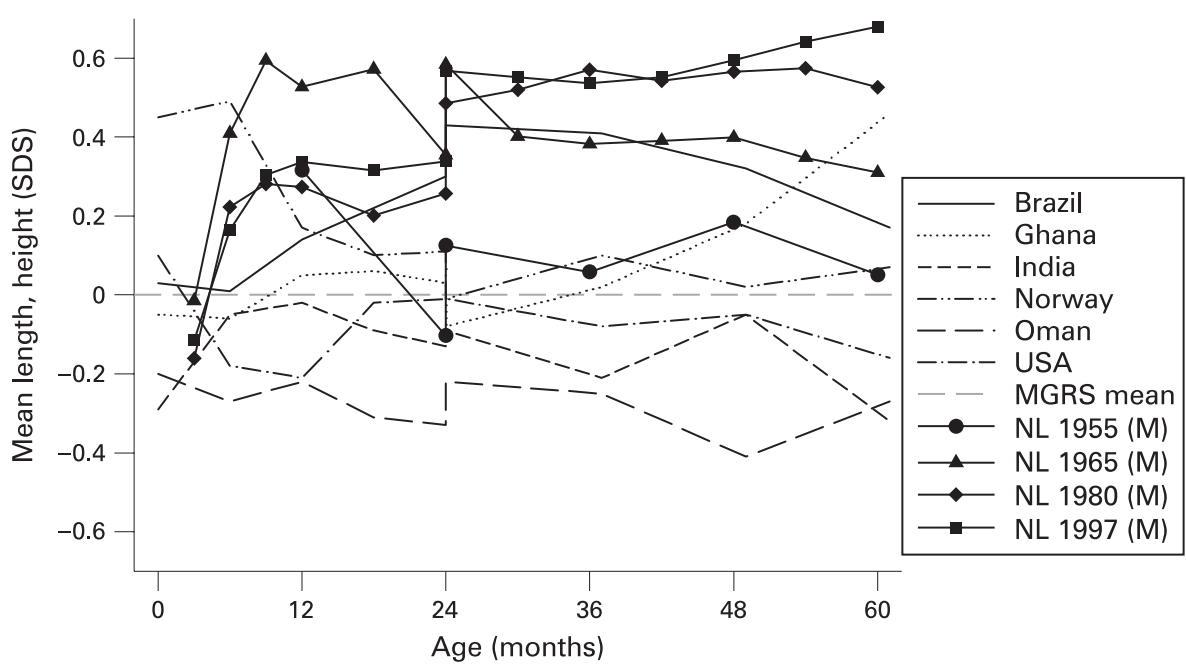

Figure 4 Mean length/height (SDS) for the six study sites from the WHO MGRS study. Data from the four Dutch growth studies from 1955, 1965, 1980 and 1997 are included.

was found to be relatively small (fig 1), pooling will increase the variance of the growth standards. Thus, individuals within a given site or population are expected to vary slightly less than the WHO-CGS. This translates into a smaller standard deviation of the SDS, typically around 0.95. The effect will increase with site variation.

\section{ADOPT OR ADAPT?}

It is sensible to explore mechanisms that may hinder adoption of the WHO-CGS. In Bangladesh, about $60 \%$ of children aged 0-5 years have a length or height below -2 SDS according to the new standards. ${ }^{13}$ Thus, most children fall into the lower region of the growth chart. On the one hand, this adequately reflects the position of these children relative to the world, and provides a strong impetus to further improve life conditions. On the other hand, for child health and clinical care, we should not expect wide acceptance of the new growth standards in Bangladesh. Health care workers, doctors and parents could perceive these standards as unattainable, not applicable to them and not relevant, and may wish to settle for a reference group that is closer to the local population.

The same applies at the other end of the spectrum. The secular increase in height of Dutch children has been documented through four nationwide growth studies since 1955. ${ }^{14}$ In 1955, the average height of Dutch boys aged 5 years was $110.2 \mathrm{~cm}$. In 1997 it was $113.1 \mathrm{~cm}$. The WHO-CGS for boys aged 5 is $109.7 \mathrm{~cm}$. In 1955, Dutch boys were already +0.05 SDS taller than the WHO-CGS and in 1997 they were +0.68 SDS taller. This translates into a difference of about $8 \mathrm{~cm}$ in average stature in adulthood. Figure 4 shows the increase between 1955 and 1997 plotted as SDS relative to the WHO-CGS. The Dutch growth charts have been updated three times since 1955 and the fifth Dutch growth study will present a new update scheduled to appear in 2009. The main argument for updating has always been to keep pace with secular changes. If this argument continues to be valid, then differences beyond a certain magnitude support the call for country-specific standards. If we were to decide that countries with a difference greater than \pm 0.5 SDS are candidates to deviate from the WHOCGS, Bangladesh and The Netherlands would qualify. For Bangladesh, the WHOCGS may encourage improvements leading to optimal growth in future generations. For the Netherlands, the WHOCGS contain a possible warning of the adverse health consequences of greater than optimal height.

The WHO-CGS do not provide information about actual growth in a given country and will not eliminate the need for prevalence studies. Given representative local data, it is relatively straightforward to estimate adaptation factors that will convert the WHO-CGS into local standards. This can even be done if age range is only partly observed. The adaptation factors will also be of interest in their own right, as they measure how much local populations differ from global standards. The challenge is to interpret and explain such differences. Moreover, the variation in adaptation factors between different countries is a measure of inequality.

To summarise, the new WHO-CGS are an extremely valuable yardstick for cross-population comparisons. The WHOCGS emphasise how children should grow. It is as yet unclear whether the new standards are applicable to a given child or a specific population. The two articles in this issue of Archives of Disease in Childhood invite the reader to consider these matters.

Competing interests: None.

Arch Dis Child 2008;93:549-551.

doi:10.1136/adc.2007.136010

\section{REFERENCES}

1. Garza C, de Onis M, for the WHO Multicentre Growth Reference Study Group. Rationale for developing a new international growth reference. Food Nutr Bull 2004;25(1 Suppl):S5-14.

2. Hui LL, Schooling CM, Cowling BJ, et al. Are universal standards for optimal infant growth appropriate? Evidence from a Hong Kong Chinese birth cohort. Arch Dis Child 2008;93:561-5.

3. Wright C, Lakshman $\mathrm{R}$, Emmett $\mathrm{P}$, et al. Implications of adopting the WHO 2006 Child Growth Standard in the UK: two prospective cohort studies. Arch Dis Child 2008;93:566-9.

4. De Onis M, Garza C, Victora CG, et al, for the Multicentre Growth Reference Study Group. The WHO Multicentre Growth Reference Study: planning, study design, and methodology. Food Nutr Bull 2004;25(1 Suppl):S15-26.

5. World Health Organization Working Group on the Growth Reference Protocol, Task Force on Methods for the Natural Regulation of Fertility Growth patterns of breastfed infants in seven countries. Acta Paediatr 2000;89:215-22.

6. WHO Multicentre Growth Reference Study Group. Assessment of differences in linear growth among populations in the WHO Multicentre Growth Reference Study. Acta Paediatr 2006;(Suppl 450):5665.

7. Kracht R. Comparison of the WHO Child Growth Standards with the Chinese 9 Cities Surveys 19751995. Abstract. Wuhan, Hubei, China: Tongji Medical College, Huazhong University of Science and Technology, 1 August 2006. Available from http:// www.troped.org/theses/thesis 85.pdf laccessed 24 April 2008)

8. Coordinating Study Group of Nine Cities on Physical Growth and Development of Children, Capital Institute of Pediatrics. [A national survey on growth of children under 7 years of age in nine cities of China, 2005] (in Chinese). Zhonghua Er Ke Za Zhi 2007:45(8):609-14.

9. Jorde LB, Wooding SP. Genetic variation, classification and 'race'. Nat Genet 2004;36/Suppl 11):S28-33

10. De Ridder M, van den Broeck J, Batstra M, et al. Association between infant growth before onset of juvenile type-1 diabetes and autoantibodies to IA-2. Lancet 2000;356:655-6.

11. Singhal A, Lucas A. Early origins of cardiovascular disease: is there a unifying hypothesis? Lancet 2004;363:1642-5

12. Herngreen WP, van Buuren S, van Wieringen JC, et al. Growth in length and weight from birth to 2 years of a representative sample of Netherlands children (born in 1988-89) related to socio-economic status and other background characteristics. Ann Hum Biol 1994;21:449-63.

13. de Onis $\mathbf{M}$, Onyango AW, Borghi $E$, et al, for the WHO Multicentre Growth Reference Study Group. Comparison of the WHO Child Growth Standards and the NCHS growth reference: implications for child health programs. Public Health Nutr 2006;9:942-7.

14. Fredriks MA, van Buuren $S$, Burgmeijer $R$, et al. Continuing positive secular growth change in The Netherlands 1955-1997. Pediatr Res 2000;47(3):316-23. 